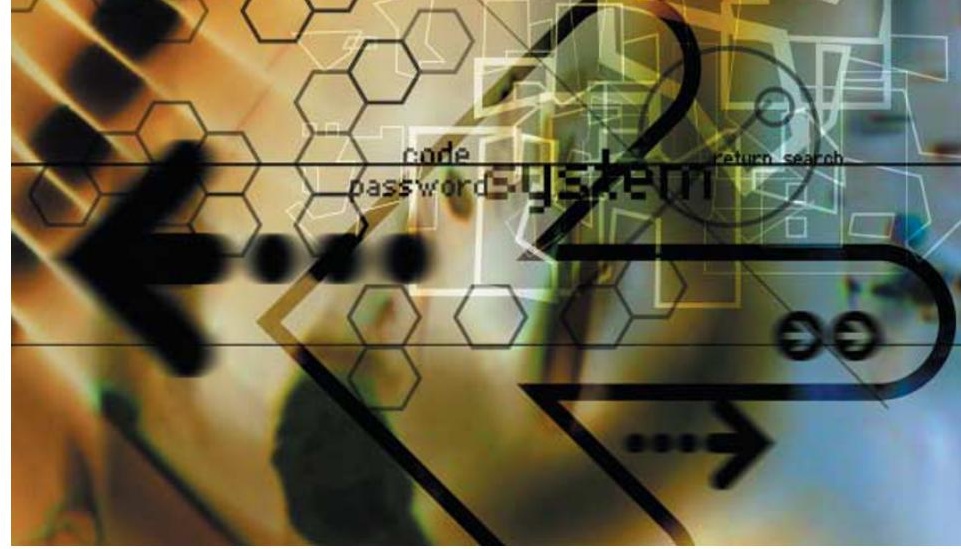

nism by which the mammalian inner ear amplifies incoming sounds now sees the publication of new evidence in favour of a mechanism driven by an influx of calcium ions into the outer hair cells. The study uses a new type of in vitro cochlear preparation that allows researchers to get closer to the process than ever before.

Writing in Nature Neuroscience, Chan and Hudspeth describe how they removed part of the cochlea of the clawed jird (also known as the Mongolian gerbil) and mounted it in a recording chamber that had two compartments. The compartments were designed to reproduce the conditions found in the inner ear, in which the apical side of the sensory epithelium is bathed in endolymph — a fluid that is rich in potassium ions — while the basal side is bathed in sodium-rich perilymph. This setup allowed the authors to record the responses of the cochlea to sound stimulation for up to 30 minutes.

The amplification process in the inner ear is an active, frequencyspecific process that optimizes the ear's response to incoming sounds. A characteristic feature of the active process is compressive nonlinearity a large range of stimulus intensities is compressed into a relatively narrow range of responses. The active process is also responsible for 'spontaneous otoacoustic emission' - sounds that are produced by the active movement of the inner hair cells and that can be recorded from the ear canal.

There are two theories about how the active process occurs. The first is active hair-bundle motility, and relies

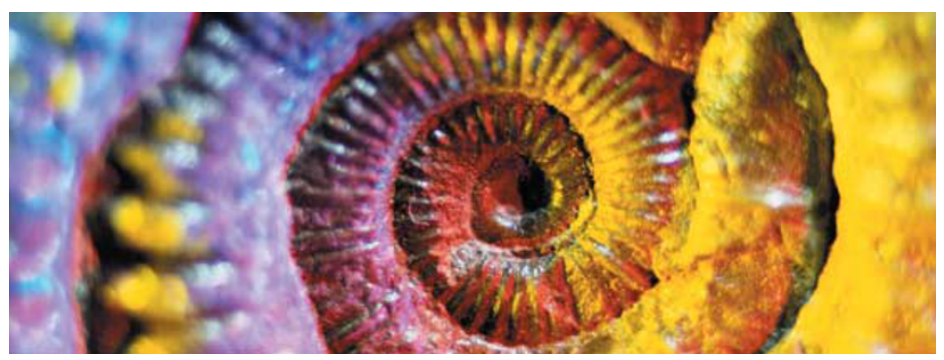

during mechanoelectrical transduction. The calcium current is thought to stimulate the closure of transduction channels and the activity of myosin motors, both of which could contribute to the amplification of the mechanical stimulation that is produced by sounds. The second theory is known as somatic electromotility. This is also initiated by electrical activity, but relies on conformational changes in a protein called prestin.

Chan and Hudspeth set out to test the first theory. They blocked the mechanoelectrical transduction channels with amiloride, so that calcium ions could not enter the cells, and found that this abolished the nonlinear amplification process. By contrast, when the ionic contents of the artificial endolymph were manipulated so that currents other than the calcium current were abolished, the nonlinear amplification persisted. These results provide evidence that calcium influx is both necessary and sufficient for the active process.

The debate will no doubt continue, with proponents of both theories continuing to test their ideas. It might be that both mechanisms are involved, perhaps at different frequencies. But the in vitro preparation used by Chan and Hudspeth should be a useful tool in the investigation.

(2) References and links ORIGINAL RESEARCH PAPER Chan, D. K. \& Hudspeth, A. J. $\mathrm{Ca}^{2+}$ current-driven nonlinear amplification by the mammalian cochlea in vitro. Nature Neurosci. 8, 149-155 (2005) WEB SITE Hudspeth Laboratory web page: http://www.rockefeller.edu/labheads/ hudspeth/hudspeth-lab.php

\section{A new direction for ALS therapy}

Amyotrophic lateral sclerosis (ALS) is a rapidly progressing degenerative disease that attacks motor neurons, leading to paralysis and death. Effective treatments for this devastating condition have evaded researchers for many years. Now, however, there is encouraging new evidence that direct delivery into the CNS of the neurotrophic factor vascular endothelial growth factor (VEGF)leads to a marked improvement in prognosis in a rat model of ALS.

The details of pathogenesis of ALS remain elusive, but Carmeliet and co-workers recently reported that low VEGF concentrations cause motor neuron degeneration that resembles ALS in mice, and are associated with an increased risk of developing ALS in humans. This points to a role for VEGF in the pathogenesis of ALS, therefore making it a potentially attractive therapeutic candidate.

Systemic delivery of VEGF is beneficial to mice with ALS, but is associated with vascular side effects and an adverse immune response. Delivery of many recombinant neurotrophic factors, such as brain-derived neurotrophic factor and ciliary neurotrophic factor, have previously been shown to prolong survival in animal models of ALS, but, so far, these successes have not been replicated in human clinical trials.

In this new study, Carmeliet and co-workers showed that direct delivery of recombinant VEGF into the cerebrospinal fluid delayed the onset of paralysis by 17 days in rats with ALS. The treatment also improved motor performance and prolonged survival by 22 days. Importantly, there were no serious adverse consequences, in contrast to previously used systemic methods of VEGF delivery.

The therapeutic success of VEGF might rest on its direct involvement in the pathogenesis of ALS. By contrast, there is only limited evidence that other neurotrophic factors are involved in motor neuron degeneration. Alternatively, the intracerebroventricular mode of delivery might allow more rapid diffusion of VEGF into the spinal cord than systemic methods.

These results represent the most promising therapeutic effect of direct protein delivery observed so far in an animal model of ALS. However, it remains to be seen whether this can be translated into an effective therapy in human clinical trials.

Alison Rowan

\section{(1) References and links}

ORIGINAL RESEARCH PAPER Storkebaum, E. et al. Treatment of motoneuron degeneration by intracerebroventricular delivery of VEGF in a rat model of ALS. Nature Neurosci. 8, 85-92 (2005)

FURTHER READING Cleveland, D. W. \& Rothstein, J. D. From Charcot to Lou Gehrig: deciphering selective motor neuron death in ALS. Nature Rev. Neurosci. 2, 806-819 (2001) 